\title{
Primary care use after cancer treatment: an analysis of linked administrative data
}

\author{
R. Urquhart $\mathrm{PhD}^{*+}$ and L. Lethbridge $\mathrm{MA}^{*}$
}

\begin{abstract}
Background Primary care-led follow-up is a safe and acceptable alternative to oncologist-led follow-up. We sought to investigate patterns of primary care use during cancer follow-up care.

Methods We identified all persons in Nova Scotia, diagnosed with an invasive breast, prostate, colorectal, or gynecologic cancer between January 2006 and December 2013. We linked this dataset to cancer centre, hospital discharge abstracts, physicians' billing, and census data. We identified a survivor cohort $(n=12,201)$, then descriptively examined primary care use during follow-up care. Multivariate Poisson and negative binomial regression, respectively, were used to examine primary care use for two outcomes: total number of primary care provider (PCP) visits (all reasons) and total number of cancer-specific PCP visits.
\end{abstract}

Results The mean numbers of PCP visits (all reasons) and cancer-specific PCP visits per year for survivors who did not receive cancer centre follow-up (CC-FUP) were 8.12 and 0.43 visits, respectively, and for survivors who continued to receive CC-FUP were 8.75 and 0.63 visits, respectively. Age, cancer type, stage at diagnosis, comorbidity scores, year of diagnosis, and receipt of CC-FUP were associated with both outcomes. Compared with prostate cancer survivors, breast, colorectal, and gynecologic cancer survivors had, respectively, 56\%, 69\%, and 56\% fewer expected cancer-specific PCP visits. Receipt of CC-FUP increased the expected number of PCP visits (all reasons) by $12 \%$ and cancer-specific PCP visits by $50 \%$.

Conclusions Primary care use was higher in survivors who continued to visit their oncology teams for follow-up. This suggests that survivors who remain with their oncology teams after treatment continue to have high needs not met by these teams alone.

Key Words Survivorship, follow-up care, administrative health data

Curr Oncol. 2020 December:27(6)e590-e595

www.current-oncology.com

\section{BACKGROUND}

Earlier detection and improved treatments have led to dramatic increases in cancer survival. Today, approximately $3 \%$ of the population are cancer survivors ${ }^{1}$, translating to nearly 1 million Canadians. The rise in prevalence has led to a recognized need to focus on survivors' care after cancer treatment, commonly referred to as follow-up care. As outlined by the U.S. Institute of Medicine, this care requires surveillance for recurrent and secondary cancers, management of late and long-term effects, and promotion of healthy behaviours to mitigate new and ongoing health concerns ${ }^{1}$. Cancer systems, however, face rising challenges to delivering optimal care for both newly diagnosed cancer patients and survivors, including increasing demand for cancer services and provider burnout or job stress ${ }^{2-7}$.
Randomized controlled trials have demonstrated that primary care-led follow-up care is safe, effective, and acceptable compared with oncologist-led care, in terms of recurrence, survival, and quality-of-life outcomes ${ }^{8-10}$. Despite the robust evidence, population-based studies continue to demonstrate that follow-up care in Canada is largely oncologist-led and poorly coordinated between specialist and primary care $^{11,12}$.

In a population-based study of breast, colorectal, gynecologic, and prostate cancer survivors diagnosed from 2006 to 2013 in Nova Scotia, we found that $37.6 \%-69.8 \%$ of survivors, depending on cancer type, were discharged to a community-based physician (for example, primary care provider [PCP] or surgeon) within 1 year of diagnosis for their follow-up care. Yet, we have limited understanding of what happens after their discharge, including whether they

Correspondence to: Robin Urquhart, Room 413, Department of Community Health and Epidemiology, Centre for Clinical Research, 5790 University Avenue, Halifax, Nova Scotia B3H 1 V7.

E-mail: robin.urquhart@nshealth.ca — DOI: https://doi.org/10.3747/co.27.5993 
visit primary care for their cancer-specific follow-up care. In this study, our objectives were to investigate patterns of primary care use during follow-up care and whether use of primary care is associated with patient or disease characteristics.

\section{METHODS}

This retrospective study used linked administrative data to investigate follow-up care for all persons diagnosed with nonmetastatic breast, colorectal, gynecologic, or prostate cancer in Nova Scotia over an 8-year period. Approval to conduct the study was granted by the Nova Scotia Health Authority's Research Ethics Board.

\section{Cohort}

Using methods previously described, ${ }^{6}$ we identified all persons in Nova Scotia diagnosed with an invasive breast, prostate, colorectal, or gynecologic cancer between January 2006 and December $2013(n=20,901)$. International Classification of Diseases for Oncology (ICD), 3rd edition, diagnosis codes were used to identify the disease sites of interest. We linked this population-based dataset, at the patient level, to the Oncology Patient Information System (OPIS), the Canadian Institute for Health Information's Discharge Abstract Database, Medical Services Insurance physicians billing data, and Canadian census data using Statistics Canada's Postal Code Conversion File. Complete data from these datasets were acquired up to 31 December 2015.

Using this dataset, we identified a survivor cohort that included only patients who had at least 1 cancer centre visit (for example, consultation or treatment). Given the nature of Nova Scotia's cancer system, individuals not visiting a cancer centre (for example, a patient receiving surgical excision only, without a medical or radiation oncologist consultation) would not visit a cancer centre for follow-up care after treatment. We then excluded those who died during the study period, those diagnosed with stage IV or metastatic disease, and those with a prior cancer diagnosis.

\section{Data Sources and Variables}

The Nova Scotia Cancer Registry (NSCR) provided patient demographics (date of birth, postal code at diagnosis), date of diagnosis, disease characteristics (histology, stage at diagnosis), prior cancer history, and date of death. Complete visit and treatment data for all cancer centres and satellite cancer clinics in the province (hereafter referred to as cancer centres) were provided by opIs. These included dates of visits, appointment types, visit duration, provider type, and centre location, resource use (that is, specific information on resources used, such as radiotherapy equipment and chemotherapy units), and recurrence.

In addition to a discrete variable available in OPIS to indicate cancer recurrence, receipt of therapy (that is, chemotherapy or radiotherapy) in the follow-up period was also presumed to be indicative of cancer recurrence. Using data from both the NSCR and OPIS, a decision rule was developed to differentiate routine follow-up visits at cancer centres from non-routine visits during the follow-up care period (for example, visits to manage complex late effects or for suspicion of recurrence). Development of this rule is described in a prior publication ${ }^{6}$.

Data on prior diagnoses was provided by the Discharge Abstract Database to compute a comorbidity count based on the list of conditions developed by Elixhauser et al. (excluding cancer-related comorbidities $)^{13}$. This was calculated for the 3-year period prior to diagnosis. Medical Services Insurance billings data provided data on PCP visits, including reasons for visits (diagnosis codes). Cancer-specific visits to PCPs were identified based on ICD-9 diagnosis codes for malignancies. The Postal Code Conversion File is a program generated by Statistics Canada to convert postal codes to census geographic areas. Using postal code data at last visit, median neighbourhood household income at the Dissemination Area level was obtained from 2011 Canadian census data. Rural and urban residence was determined based on postal code at last visit, with a second digit of 0 indicating rural residence.

\section{Analysis}

The follow-up care period was defined as starting 1 year after the diagnosis date and continuing until the person was censored or the end of the study period was reached. This definition is consistent with definitions in earlier studies using administrative data to examine cancer follow-up practices ${ }^{14-17}$, where the follow-up period commenced 1 year after diagnosis to exclude tests and visits associated with postoperative complications, routine postoperative visits, and adjuvant therapy. Survivors were censored on evidence of a new primary cancer, cancer recurrence, or death.

Descriptive statistics were computed to describe primary care use by follow-up year, disease site, and receipt of any cancer centre follow-up care [CC-FUP (yes or no)]. Multivariate regression was used to examine primary care use, adjusting for other covariates, for 2 outcomes: total number of PCP visits (all reasons) and total number of cancer-specific PCP visits. Specifically, Poisson regression was used to model the total number of PCP visits per year during follow-up, and, to account for the overdispersion of zeros, negative binomial regression was used to model the total number of cancer-specific PCP visits per year during follow-up. Because the time period available for follow-up visits was shorter for individuals diagnosed later in the study period and the number of visits was expected to decrease over time, leading to a smaller average number of visits per year for those diagnosed later, an offset technique was used to normalize visits to exposure time (that is, the time from diagnosis until censoring or the end of the study). Regression coefficients were converted to incidence rate ratios (IRRs) that, in this context, can be interpreted as the difference in primary care visits per period of exposure. Using this approach, results are relative to the reference category: men for sex, prostate cancer survivors for disease site, stage I disease for stage at diagnosis, and urban residence for place of residence. Models were run with the following covariates: year of diagnosis, CC-FUP (yes or no), age at diagnosis (in years), sex, disease site, stage at diagnosis (I, II, III, unknown), comorbidity score, neighbourhood median household income, and place of 
residence (rural or urban). To test our definition of ongoing cancer centre follow-up ( $\geq 1$ routine follow-up visits), we conducted a sensitivity analysis with the definition being 2 or more routine follow-up visits.

\section{RESULTS}

The final cohort included 12,201 cancer survivors. Table I presents demographic data for survivors who did not receive any CC-FUP and survivors who received at least 1 CC-FUP visit. The mean number of PCP visits (all reasons) per year for survivors who did not receive CC-FUP and survivors who continued to receive CC-FUP was 8.12 and 8.75 visits respectively. The total number of cancer-specific PCP visits per year for survivors who did not receive CC-FUP and for survivors who continued to receive CC-FUP was 0.45 and 0.63 visits respectively. Table II presents the mean and median number of all PCP visits by follow-up year. For each year, there were no significant differences between survivors who did not receive CC-FUP and those who continued to receive CC-FUP. As expected, however, the number of PCP visits declined as years since diagnosis increased. Table III presents the mean and median numbers of all PCP visits per year and total number of cancer-specific PCP visits per year by disease site. For all PCP visits, only prostate cancer survivors had a significantly different mean number of visits between those who did not receive CC-FUP and those who continued to receive CC-FUP. For cancer-specific PCP visits, breast, colorectal, and prostate cancer survivors had a significantly different mean number of visits between these two groups.

Table IV presents factors associated with PCP use. Compared with prostate cancer survivors, survivors of colorectal and gynecologic cancers had 5\% and 7\% fewer PCP visits (all reasons), respectively, while survivors of breast, colorectal, and gynecologic cancers had $56 \%$ [IRR: 0.44; $95 \%$ confidence interval (CI): 0.35 to 0.55 ), $69 \%$ (IRR: $0.31 ; 95 \%$ CI: 0.27 to 0.36 ), and $56 \%$ (IRR: $0.44 ; 95 \%$ CI: 0.34 to 0.56 ) fewer cancer-specific PCP visits respectively. Compared with men, women had $13 \%$ increased risk of all PCP visits (IRR: 1.13; 95\% CI: 1.10 to 1.16), yet no difference in risk of cancer-specific PCP visits. For both outcomes, patients with a higher stage at diagnosis and comorbidity scores had higher risk of PCP use.

Year of diagnosis was associated with having an increased number of PCP visits (all reasons and cancerspecific). Specifically, each successive diagnosis (calendar) year was associated with a $29 \%$ increase in all PCP visits (IRR: 1.29 ; 95\% CI:1.28 to 1.29 ) and a $39 \%$ increase in cancer-specific PCP visits (IRR: $1.39 ; 95 \%$ CI: 1.36 to 1.41 ). This means that survivors diagnosed later in the study period were expected to have $29 \%$ and $39 \%$ more PCP visits and cancer-specific PCP visits per year respectively. Receipt of any routine CC-FUP increased the risk of all PCP visits by $12 \%$ (IRR: 1.12; 95\% CI:1.11 to 1.14) and cancer-specific PCP visits by $50 \%$ (IRR: 1.50 ; $95 \%$ CI: 1.38 to 1.63 ). The findings of the sensitivity analysis ( $\geq 2$ follow-up visits) were unchanged from the main analysis.

\section{DISCUSSION}

In a population-based cohort of cancer survivors diagnosed over an 8-year time period, this study examined primary care use during follow-up care and whether use of primary care is associated with certain characteristics. We found primary care use (both all reasons and

TABLE I Survivor characteristics

\begin{tabular}{|c|c|c|c|c|}
\hline \multirow[t]{3}{*}{ Variable } & \multicolumn{4}{|c|}{ Cancer centre follow-up care } \\
\hline & \multicolumn{2}{|c|}{ No } & \multicolumn{2}{|c|}{ Yes $^{\mathrm{a}}$} \\
\hline & Value & $95 \% \mathrm{Cl}$ & Value & $95 \% \mathrm{Cl}$ \\
\hline Survivors (n) & 7458 & & 4743 & \\
\hline Mean age (years) & $63.8 \pm 11.7$ & 63.5 to 64.1 & $61.0 \pm 11.3$ & 60.7 to 61.3 \\
\hline Women (\%) & 47.8 & 46.7 to 49.0 & 63.9 & 62.5 to 65.2 \\
\hline \multicolumn{5}{|l|}{ Cancer type (\%) } \\
\hline Breast & 28.1 & 27.1 to 29.1 & 45.3 & 43.9 to 46.7 \\
\hline Colorectal & 25.8 & 24.8 to 26.8 & 11.6 & 10.7 to 12.5 \\
\hline Gynecologic & 7.7 & 7.1 to 8.3 & 13.2 & 12.2 to 14.2 \\
\hline Prostate & 38.4 & 37.3 to 39.5 & 29.9 & 28.6 to 31.2 \\
\hline \multicolumn{5}{|l|}{ Stage at Dx (\%) } \\
\hline I & 34.3 & 33.2 to 35.3 & 30.2 & 28.9 to 31.5 \\
\hline II & 41.1 & 40.0 to 42.2 & 41.2 & 39.8 to 42.7 \\
\hline III & 13.4 & 12.7 to 14.2 & 21.4 & 20.2 to 22.5 \\
\hline Unknown & 11.2 & 10.5 to 11.9 & 7.2 & 6.5 to 7.9 \\
\hline Mean comorbidity score & $0.62 \pm 0.95$ & 0.60 to 0.64 & $0.52 \pm 0.81$ & 0.50 to 0.55 \\
\hline Mean income (\$) & $59,450 \pm 21,472$ & 58,960 to 59,941 & $59,743 \pm 20,750$ & 59,148 to 60,338 \\
\hline Rural residence (\%) & 36.1 & 35.0 to 37.1 & 31.2 & 29.9 to 32.5 \\
\hline
\end{tabular}

a One or more cancer centre follow-up visits.

$\mathrm{Cl}=$ confidence interval; $\mathrm{Dx}=$ diagnosis. 
TABLE II Primary care provider visits (all reasons) by follow-up year

\begin{tabular}{lccccc}
\hline \multirow{2}{*}{$\begin{array}{c}\text { Follow-up } \\
\text { year }\end{array}$} & \multicolumn{3}{c}{ Cancer centre follow-up care } \\
\cline { 2 - 3 } \cline { 6 - 6 } \cline { 5 - 6 } & Mean & Median (IQR) & & Mean & Median (IQR) \\
\hline 1 & 8.90 & $7(7)$ & & 9.09 & $7(8)$ \\
\hline 2 & 8.04 & $6(7)$ & & 8.34 & $7(7)$ \\
\hline 3 & 6.84 & $5(9)$ & & 6.85 & $5(8)$ \\
\hline 4 & 5.66 & $3(8)$ & & 5.68 & $4(8)$ \\
\hline 5 & 4.60 & $1(7)$ & & 4.67 & $2(7)$ \\
\hline
\end{tabular}

a One or more cancer centre follow-up visits.

$\mathrm{IQR}=$ interquartile range. cancer-specific reasons) was higher in survivors who continued to see their oncology teams for routine follow-up. We further found that many patient, disease, and system factors are associated with PCP use. Several of these factors (for example, stage at diagnosis, presence of comorbidities) might be expected to drive PCP use, given a higher severity of disease and symptoms or conditions requiring ongoing management. Others (for example, disease site, receipt of CC-FUP) are perhaps unanticipated or more difficult to explain. Our findings are novel and help identify subpopulations where further investigation is needed.

That survivors who continue to receive routine CCFUP have higher PCP use during the follow-up care period than those who have transitioned to the community is an

TABLE III Primary care provider use per year by disease site ${ }^{a}$

\begin{tabular}{|c|c|c|c|c|c|c|c|c|}
\hline \multirow[t]{3}{*}{ Visit type } & \multicolumn{8}{|c|}{ Cancer centre follow-up care } \\
\hline & \multicolumn{2}{|c|}{ Breast } & \multicolumn{2}{|c|}{ Colorectal } & \multicolumn{2}{|c|}{ Gynecologic } & \multicolumn{2}{|l|}{ Prostate } \\
\hline & No & Yes $^{b}$ & No & Yes $^{b}$ & No & Yes $^{b}$ & No & Yes $^{b}$ \\
\hline \multicolumn{9}{|l|}{ All, per year } \\
\hline Mean & $8.71 \pm 7.38$ & $8.73 \pm 6.79$ & $8.68 \pm 7.82$ & $9.25 \pm 7.99$ & $7.40 \pm 10.42$ & $8.11 \pm 7.86$ & $7.46 \pm 6.81$ & $8.87 \pm 7.08$ \\
\hline Median (IQR) & $7(7)$ & $7(6)$ & $7(7)$ & $7(8)$ & $6(6)$ & $6(7)$ & $6(7)$ & $7(6)$ \\
\hline \multicolumn{9}{|c|}{ Cancer-specific, per year } \\
\hline Mean & $0.27(0.73)$ & $0.39(1.44)$ & $0.26(0.96)$ & $0.38(0.97)$ & $0.26(1.55)$ & $0.38(1.18)$ & $0.74(1.41)$ & $1.20(1.57)$ \\
\hline Median (IQR) & $0(0)$ & $0(0)$ & $0(0)$ & $0(0)$ & $0(0)$ & $0(0)$ & $0(1)$ & $1(2)$ \\
\hline
\end{tabular}

a Boldface type indicates statistical significance at the $95 \%$ confidence level.

b One or more cancer centre follow-up visits.

$\mathrm{IQR}=$ interquartile range.

TABLE IV Factors associated with primary care use during follow-up care ${ }^{a}$

\begin{tabular}{|c|c|c|c|c|}
\hline \multirow[t]{3}{*}{ Factor } & \multicolumn{4}{|c|}{ Total primary care provider visits per year } \\
\hline & \multicolumn{2}{|c|}{ All reasons } & \multicolumn{2}{|c|}{ Cancer-specific } \\
\hline & IRR & $95 \% \mathrm{Cl}$ & IRR & $95 \% \mathrm{Cl}$ \\
\hline Year of Dx & 1.29 & 1.28 to 1.29 & 1.39 & 1.36 to 1.41 \\
\hline Received cancer centre follow-up care ${ }^{b}$ & 1.12 & 1.11 to 1.14 & 1.50 & 1.38 to 1.63 \\
\hline Age & 1.02 & 1.02 to 1.02 & 1.01 & 1.00 to 1.01 \\
\hline Women & 1.13 & 1.10 to 1.16 & 0.86 & 0.71 to 1.05 \\
\hline \multicolumn{5}{|l|}{ Cancer site ${ }^{\mathrm{c}}$} \\
\hline Colorectal & 0.95 & 0.93 to 0.97 & 0.31 & 0.27 to 0.36 \\
\hline Breast & 1.03 & 1.00 to 1.07 & 0.44 & 0.35 to 0.55 \\
\hline Gynecologic & 0.93 & 0.90 to 0.97 & 0.44 & 0.34 to 0.56 \\
\hline \multicolumn{5}{|l|}{ Disease stage $^{d}$} \\
\hline II & 1.00 & 0.99 to 1.02 & 1.25 & 1.13 to 1.38 \\
\hline III & 1.04 & 1.02 to 1.06 & 1.42 & 1.25 to 1.60 \\
\hline Unknown & 0.99 & 0.96 to 1.01 & 1.10 & 0.94 to 1.28 \\
\hline Comorbidity score & 1.12 & 1.11 to 1.13 & 1.07 & 1.03 to 1.12 \\
\hline Income & 1.00 & 1.00 to 1.00 & 1.00 & 1.00 to 1.00 \\
\hline Rural residence & 0.96 & 0.95 to 0.98 & 1.05 & 0.97 to 1.15 \\
\hline
\end{tabular}

a Boldface type indicates statistical significance at the 95\% confidence level.

b One or more cancer centre follow-up visits.

c Compared with survivors of prostate cancer.

d Compared with stage I.

$\mathrm{IRR}=$ incidence rate ratio; $\mathrm{Cl}=$ confidence interval; $\mathrm{Dx}=$ diagnosis. 
important finding that requires attention. In our prior work on this same cohort, we found approximately half of these survivors were discharged back to the community after their treatment for follow-up care. While many patient, provider, and system factors undoubtedly influence decisions around follow-up care, oncologists ultimately make these decisions, including whether survivors are discharged back to primary care. The findings from this study may point to a situation where oncologists are implicitly risk-stratifying survivors, presumably based on their risk of recurrence and other adverse late or long-term effects, and transitioning the survivors they deem low-risk while continuing to see those they deem at higher risk. Following this explanation, the data may show that survivors who were transitioned (or deemed "low-risk") had fewer needs, whether physical or psychosocial in nature, and therefore visited their PCPs less than those with higher needs. Conversely, survivors who were not transitioned (or deemed "higher-risk") had higher needs, which could not be met by seeing their oncology teams alone and therefore had higher concomitant use of PCPs. While this is just one interpretation of our findings, it has been discussed with oncologists in Nova Scotia and resonates with their experience. Recent publications support the concept of using a risk stratification approach to provide a more personalized model of follow-up care for individual survivors ${ }^{18-20}$. Future research is needed to develop ways to systematically risk-stratify survivors and identify those suitable for transition back to community care. This should involve not only consideration of disease characteristics (for example, risk of recurrence) but also the various patient, clinical, and contextual factors that oncologists consider when making follow-up care decisions.

Survivors of prostate cancer visited PCPs for cancerspecific concerns more often than survivors of breast, colorectal, or gynecologic cancers, regardless of whether they received follow-up care from cancer centres. Moreover, those receiving CC-FUP visited their PCPs even more than those not receiving CC-FUP. While the data do not point to reasons for this, the differences in risk compared with survivors of other cancers are striking (see Table IV). No Canadian data related to routine follow-up care or PCP use during the follow-up period are available for prostate cancer survivors with which to compare the present findings. Nevertheless, there are several explanations for these findings. It is possible that men are not having all of their post-treatment needs met by their cancer care team, that they have greater trust in their PCP to discuss and manage disease- and treatment-related long-term effects (for example, impaired urinary, bowel, and sexual function), and/or that poor coordination of care is leading to a lack of role clarity and duplication of cancer follow-up care (for example, prostate-specific antigen testing). Research has found that prostate cancer survivors rate their PCPs higher than their oncologists on items related to coordination of care, comprehensive care, and strength of relationship, and that they view their PCPs more positively than do breast cancer survivors on the strength of their PCP relationship ${ }^{21}$. Another study found that prostate cancer survivors prefer to receive sexual and emotional health information from their PCPs ${ }^{22}$. Future research should explore these hypotheses further to ensure follow-up care is delivered in ways that support prostate cancer survivors and optimize patient and system outcomes.

Strengths of this study include examination of a large population-based cohort of survivors diagnosed over an 8-year time period and use of a more precise measure of routine CC-FUP visits than that used in prior research. Regarding the latter, researchers investigating follow-up care in Canada have typically quantified all visits to oncologists during the follow-up period as "routine" follow-up care, whereas we developed a decision rule, using a combination of NSCR and OPIS data, to differentiate routine from non-routine visits during the follow-up care period ${ }^{6}$.

There are also a number of limitations. First, this study was conducted in one Canadian province only, and it is unclear whether and how primary care use during follow-up would be similar elsewhere in Canada. Further study is needed in other Canadian jurisdictions to better understand primary care use after treatment and to help inform risk-stratified models of follow-up care. A second limitation relates to the nature of administrative data, which are routinely collected in the course of care activities and not for research purposes. Thus, the data might not be as reliable as desired for research. For example, cancer-specific PCP visits are likely underestimated, given that if a survivor discusses issues related to a prior cancer diagnosis during a visit to their PCP for another health issue, the billings claim that is submitted might only reflect the health issue that was the main purpose of the visit. A third limitation is the dichotomous categorization of primary care visits as either a cancer-related visit or non-cancer-related visit. While a further breakdown of non-cancer primary care use would be informative, we did not go this route in this study for various reasons, including the limited reliability of primary care billing diagnosis codes as well as small cell sizes. Fourth, we were unable to ascertain whether individuals with higher primary care use during survivorship also had higher primary care use before their cancer diagnosis. A recent study examining primary care use in breast cancer patients across the continuum of care found that high use of primary care pre-cancer diagnosis was associated with high use of primary care during all phases of cancer care: diagnosis, treatment, and survivorship ${ }^{23}$. Finally, our reliance on hospitalization data to compute a comorbidity score is a conservative approach and therefore we could have missed less-severe conditions or those managed by a family physician. However, diagnoses recorded in the Discharge Abstract Database are either the cause of the hospital admission, or they affected resource consumption or length of stay during the hospital admission. Thus, these diagnoses are more likely to be definitive diagnoses and are potentially more severe, with a greater impact on a person's life and health care use. We also calculated the comorbidity score using 3 years of pre-diagnosis data rather than the typical 1-year lookback period, allowing for a more comprehensive calculation.

\section{CONCLUSIONS}

The findings of this study demonstrate that survivors who continue to receive CC-FUP care visit their PCP (both all reasons and cancer-specific reasons) more than those 
discharged to the community. This may suggest that oncologists implicitly apply a risk-stratified approach to discharge survivors they perceive to be at lower risk or to have lower needs after treatment. Future research is needed to develop and test risk-stratified models of follow-up care, including evidence-informed tools, to help oncology teams systematically identify who to transition back to primary care and models of shared care to ensure coordinated, quality follow-up for the subpopulation of patients who continue to see oncology for their care.

\section{ACKNOWLEDGMENTS}

We thank Cynthia Kendell and Margaret Jorgensen (research coordinators), who assisted with ethics approval and data access. This study was funded by a grant from the Nova Scotia Health Authority Research Fund.

\section{CONFLICT OF INTEREST DISCLOSURES}

We have read and understood Current Oncology's policy on disclosing conflicts of interest, and we declare that we have none.

\section{AUTHOR AFFILIATIONS}

*Department of Community Health and Epidemiology, Dalhousie University, and ${ }^{\dagger}$ Nova Scotia Health Authority, Halifax, NS.

\section{REFERENCES}

1. Hewitt ME, Greenfield S, Stovall E, eds. From Cancer Patient to Cancer Survivor: Lost in Transition Washington, DC: National Academies Press; 2006.

2. Grunfeld E, Earle CC. The interface between primary and oncology specialty care: treatment through survivorship. J Natl Cancer Inst Monogr 2010;2010:25-30.

3. Kendell C, Decker KM, Groome PA, et al. Use of physician services during the survivorship phase: a multi-province study of women diagnosed with breast cancer. Curr Oncol2017;24:81-9.

4. Earle CC. Long-term care planning for cancer survivors: a health services research agenda.JCancer Surviv 2007;1:64-74.

5. Chubak J, Tuzzio L, Hsu C, et al. Providing care for cancer survivors in integrated health care delivery systems: practices, challenges, and research opportunities. J Oncol Pract 2012;8:184-9.

6. Urquhart R, Lethbridge L, Porter GA. Patterns of cancer centre follow-up care for survivors of breast, colorectal, gynecologic, and prostate cancer. Curr Oncol 2017;24:360-6.

7. Grunfeld E, Zitzelsberger L, Coristine M, Whelan TJ, Aspelund F, Evans WK. Job stress and job satisfaction of cancer care workers. Psychooncology 2005;14:61-9.

8. Grunfeld E, Mant D, Yudkin P, et al. Routine follow-up of breast cancer in primary care: a randomised trial. $B M J$ 1996;313:665-9.

9. Grunfeld E, Levine MN, Julian JA, et al. Randomized trial of long-term follow-up for early-stage breast cancer: a comparison of family physician versus specialist care. J Clin Oncol 2006;24:848-55.

10. Wattchow DA, Weller DP, Esterman A, et al. General practice vs surgical-based follow-up for patients with colon cancer: randomised controlled trial. Br J Cancer 2006;94:1116-21.

11. Kendell C, Decker KM, Groome PA, et al. Use of physician services during the survivorship phase: a multi-province study of women diagnosed with breast cancer. Curr Oncol 2017; 24:81-9.

12. Urquhart R, Lethbridge L, Porter G. Patterns of cancer centre follow-up care for survivors of breast, colorectal, gynecologic, and prostate cancer. Curr Oncol 2017;24:360-6.

13. Elixhauser A, Steiner C, Harris DR, Coffey RM. Comorbidity measures for use with administrative data. Med Care 1998; $36: 8-27$.

14. Urquhart R, Folkes A, Porter G, et al. Population-based longitudinal study of follow-up care for colorectal cancer patients in Nova Scotia. J Oncol Pract 2012;8:246-52.

15. Grunfeld E, Hodgson DC, Del Giudice ME, Moineddin R. Population-based longitudinal study of follow-up care for breast cancer survivors. J Oncol Pract 2010;6:174-81.

16. Snyder CF, Earle CC, Herbert RJ, Neville BA, Blackford AL, Frick KD. Trends in follow-up and preventive care for colorectal cancer survivors. J Gen Intern Med 2008;23:254-9.

17. Snyder CF, Frick KD, Kantsiper ME, et al. Prevention, screening, and surveillance care for breast cancer survivors compared with controls: changes from 1998 to 2002. J Clin Oncol 2009;27:1054-61.

18. Oeffinger KC, McCabe MS. Models for delivering survivorship care. J Clin Oncol 2006;24:5117-24.

19. Taplin SH, Brown Rodgers A. Toward improving the quality of cancer care: addressing the interfaces of primary and oncology-related subspecialty care. J Natl Cancer Inst Monogr 2010;2010:3-10.

20. Watson EK, Rose PW, Neal RD, et al. Personalised cancer follow-up: risk stratification, needs assessment or both? Br J Cancer 2012;106:1-5.

21. Hudson SV, Ohman-Strickland PA, Bator A, et al. Breast and prostate cancer survivors' experiences of patient-centered cancer follow-up care from primary care physicians and oncologists. J Cancer Surviv 2016;10:906-14.

22. Zhou ES, Bober SL, Nekhlyudov L, Hu JC, Kantoff PW, Recklitis CJ. Physical and emotional health information needs and preferences of long-term prostate cancer survivors. Patient Educ Couns 2016;99:2049-54.

23. Decker K, Moineddin R, Kendell C, et al. on behalf of the Canadian Team to Improve Community-Based Cancer Care Along the Continuum (CanIMPACT). Changes in primary care provider utilization by phase of care for women diagnosed with breast cancer: a CanIMPACT longitudinal cohort study. BMC Fam Pract 2019;20:161. 